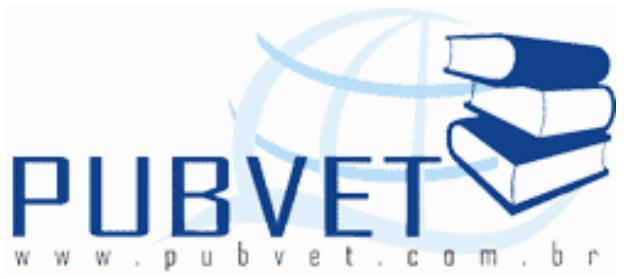

PUBVET, Publicações em Medicina Veterinária e Zootecnia.

\title{
Fator inseminador num protocolo de inseminação artificial em tempo fixo: relato de caso
}

\author{
Gabriela Lucia Bonato
}

Graduada e mestranda pela Universidade Federal de Uberlândia

\section{Resumo}

Objetivou-se com este estudo, relatar a importância de um bom inseminador dentro de um programa de inseminação artificial em tempo fixo (IATF) durante os meses de setembro a novembro de 2010 utilizando 29 vacas leiteiras mestiças divididas em três lotes que seguiram um mesmo protocolo: D0 implante de progesterona com benzoato de estradiol, D8 - retirada do implante, prostaglandina e ECG, D9 (manhã) - benzoato de estradiol, D9 (tarde) - GNRH e D10 - IATF. O lote um $(n=10)$ foi inseminado em setembro pelo inseminador da fazenda. O lote dois $(n=10)$ foi inseminado pelo mesmo inseminador no mês de outubro, e no lote três foram inseminados 9 animais por um inseminador experiente no mês de novembro. Os índices de prenhez foram $10 \%(1 / 10) ; 20 \%(2 / 10)$ e $77,77 \%$ (7/9) respectivamente para os lotes um, dois e três, podendo confirmar o quanto a mão-de-obra treinada é importante para obtenção de bons resultados em programas reprodutivos.

Palavras-chave: vacas mestiças, prenhez, controle reprodutivo. 


\title{
Inseminator factor in an artificial insemination protocol in timed artificial insemination: case report
}

\begin{abstract}
The objective of this study, we report the importance of a good artificial insemination program within a fixed time artificial insemination (TAI) during the months September to November 2010 using 29 crossbred dairy cows were divided into three lots that have followed the same Protocol: D0-implant of progesterone with estradiol benzoate, D8 - implant removal, prostaglandin and ECG, D9 (morning) - estradiol benzoate, D9 (afternoon) - GnRH and D10 - TAI. Lot one ( $n=10)$ was inseminated in September by artificial insemination of the farm. Lot two $(n=10)$ was inseminated by the same artificial insemination in October, and three in the batch were inseminated by a nine animals inseminator experienced in November. The pregnancy rates were $10 \%$ (1/10) 20\% (2 / 10) and 77.77\% (7 / 9) respectively for lots one, two and three, and can confirm how much manpower trained is important for obtaining good results in reproductive programs.
\end{abstract}

Keywords: crossbred cows, pregnancy, reproductive control.

\section{Introdução}

Apesar das grandes dimensões continentais brasileiras e de possuir um dos maiores rebanhos bovinos do mundo, a produtividade leiteira média no Brasil, quando comparada a outros países, não se apresenta compatível com a magnitude de seu potencial.

Segundo Zoccal (1997), a produção de leite aumentou em 1975 de 5.513 milhões de litros de leite para 17.931 milhões em 1995. Esse aumento, porém é acompanhado do crescente número de vacas ordenhadas de 11.080 cabeças para 13.722 respectivamente.

Dessa forma, um rebanho leiteiro deve ter um controle reprodutivo a fim de evitar altos intervalos de partos e conseqüentemente aumento nas 
BONATO, G.L. Fator inseminador num protocolo de inseminação artificial em tempo fixo: relato de caso. PUBVET, Londrina, V. 5, N. 23, Ed. 170, Art. 1144, 2011.

despesas com vacas secas e descarte de animais (LOPEZ-GATIUS et al., 2005). A utilização da inseminação artificial (IA) é uma biotecnologia simples, de fácil acesso e baixo custo, porém até hoje o seu gargalo é caracterizado pelas falhas na detecção visual do estro, seja pela mão-de-obra pouco capacitada. Senger (1994) apontou uma eficiência de detecção visual de estro menor ou igual a $50 \%$ na maioria das fazendas leiteiras.

A inseminação artificial em tempo fixo (IATF) é outra tecnologia que possibilita solucionar as dificuldades da IA convencional, pois elimina a observação de estro e encurta o anestro pós-parto, principais responsáveis pela baixa taxa de serviço e prenhez dos programas de IA tradicionais. Pursley et al. (1997) relataram um menor impacto da baixa eficiência de detecção de estro em vacas lactantes obtido com uso de protocolos de sincronização da ovulação e inseminação artificial em tempo fixo (IATF).

Os fatores mais importantes para que esta biotecnologia produza resultados satisfatórios são: condição nutricional e sanitária adequada do rebanho, utilização de sêmen de boa qualidade, infra-estrutura, manejo adequado dos fármacos e dos animais e experiência na técnica de inseminação (GOFERT, 2008).

Objetivou-se com este trabalho avaliar o efeito do inseminador num programa de IATF em vacas leiteiras mestiças.

\section{Material e Métodos}

A pesquisa foi realizada na Estância Verônica, pertencente ao município de Ipameri-GO. As vacas receberam silagem de milho e a suplementação concentrada foi administrada diariamente durante as ordenhas, de acordo com a produção individual de cada vaca.

Foram utilizadas 29 vacas leiteiras mestiças em lactação, com mais de 45 dias pós-parto, diagnosticadas vazias e com escore de condição corporal acima de 2,50 na avaliação pré-protocolo. Elas foram divididas em lote 1 $(n=10)$ e foram inseminadas pelo funcionário da fazenda, lote $2(n=10)$, 
BONATO, G.L. Fator inseminador num protocolo de inseminação artificial em tempo fixo: relato de caso. PUBVET, Londrina, V. 5, N. 23, Ed. 170, Art. 1144, 2011.

inseminadas pelo mesmo funcionário e lote $3(n=9)$ que foram inseminadas por um inseminador experiente. Todas seguiram um mesmo protocolo hormonal: D0 - implante de progesterona com benzoato de estradiol, D8 retirada do implante, prostaglandina e ECG, D9 (manhã) - benzoato de estradiol, D9 (tarde) - GNRH e D10 - IATF.

É importante ressaltar que as inseminações realizadas pelo funcionário da fazenda eram demoradas e que muitas vezes causada lesões no aparelho reprodutivo das fêmeas devido a presença de rajas de sangue encontradas no aplicador no final da técnica.

O dignóstico de prenhez foi realizado aos 30 dias com auxílio de um ultrasson (Mindray $\left.{ }^{\circ} D P 3300\right)$. A resposta ao protocolo foi calculada diante da percentagem da taxa de prenhez.

\section{Resultados e Discussão}

O lote um, dois e três obtiveram uma taxa de gestação de $10 \%(1 / 10)$; $20 \%(2 / 10)$ e $77,77 \%$ (7/9) respectivamente. Um estudo realizado por Inforzato e colaboradores (2008) relataram uma taxa de prenhez de 50\% porém utilizando outro protocolo e em novilhas guzerá. Outros estudos como o de Barbosa (2009) que usaram um protocolo descrito por Cardoso (2006) em vacas mestiças leiteiras relataram uma concepção de 35,71\%.

O efeito de protocolo não teve variação, já que foi utilizado o mesmo nos três lotes. Já o efeito mão-de-obra é de extrema importância dentro de um programa de inseminação, pois determina o local correto de deposição do sêmen além de traumas ou lesões no aparelho reprodutivo causados por inseminadores inexperientes. Neste contexto, o presente estudo corrobora com o de Costa e Silva (2007) que confirma que as pessoas responsáveis pelo manejo com o gado devem receber treinamento específico a fim de entenderem o mecanismo animal.

Existe o fator estresse térmico durante as diferentes épocas do ano, que também pode ser o responsável pelas grandes diferenças de concepção 
BONATO, G.L. Fator inseminador num protocolo de inseminação artificial em tempo fixo: relato de caso. PUBVET, Londrina, V. 5, N. 23, Ed. 170, Art. 1144, 2011.

encontradas no presente estudo, o que concorda com Barbosa (2009), que percebeu que vacas mestiças tiveram maior concepção nos meses de outono e inverno (40 e 41,8\%) respectivamente e menor nos meses de primavera e verão $(27,7$ e $20,8 \%)$.

\section{Conclusão}

O efeito positivo da mão-de-obra capacitada é de essencial importância dentro de programas reprodutivos.

\section{Referências}

BARBOSA, C. F. Inseminação artificial em tempo fixo e diagnóstico precoce de gestação em vacas leiteiras mestiças. 2009. 41f. Dissertação (Mestrado em Ciências Veterinárias) - Universidade Federal de Uberlândia, Uberlândia, 2009.

CARDOSO, B. L.; PESCARA, J. B.; VASCONCELOS, J. L. M. Protocolo de inseminação artificial em tempo fixo para vacas mestiças leiteiras. Acta Scientiae Veterinariae, v.34, suppl. 1, p.428 (Abstr.), 2006.

COSTA E SILVA, E. V. Comportamento e eficiência reprodutiva. Revista Brasileira de Reprodução Animal, Belo Horizonte, v.31, n.2, p.177-182, 2007.

Gofert, L. F. Programas de inseminação artificial em tempo fixo (IATF). Aspectos técnicos e econômicos. In: SIMPÓSIO DE REPRODUÇÃO DE BOVINOS, 1., 2008, Pelotas. Anais...Pelotas: Embrapa, 2008. p.41-47.

INFORZATO, G. R.; SANTOS, W. R. M.; CLIMENI, B. S.O. et al. Emprego de IATF (Inseminação artificial em tempo fixo) como alternativa na reprodução da pecuária de corte. Revista Científica Eletrônica de Medicina Veterinária, Garça, ano VI, n.11, periódico semestral, 2008.

LOPEZ-GATIUS, F. et al. Ovulation failure and double ovulation in dairy cattle: risks factors and effects. Theriogenology, New York, v. 63, p. 1298-1307, 2005. Disponível em:

<http://www.sciencedirect.com/science?_ob=ArticleURL\&_udi=B6TCM-4D4D6DJ-

$5 \&$ _user $=687360 \&$ _coverDate $=03 \% 2 F 15 \% 2 F 2005 \&$ rdoc $=1 \&$ fmt $=$ high\&_orig $=$ search \&_sort $=\mathrm{d} \& \_$docanchor $=\& \mathrm{view}=\mathrm{c} \& \_$searchStrid $=1363438999 \&$ rerunO $\operatorname{rigin}=$ scholer.google\&_acct $=\mathrm{CO}$ 00037900\&version $=1 \&$ \&urlVersion $=0$ \&_userd $=687360 \&$ md5 $=00017 \mathrm{~b} 6 \mathrm{~b} 14 \mathrm{~b} 320586 \mathrm{~b} 1223 \mathrm{fbc} 29$ 79905>. Acesso em: 30 mar. 2010.

PURSLEY, J.R.; WILTBANK, M.C.; STEVENSON, J.S. et al. Pregnancy rates per artificial insemination for cows and heifers inseminated at a synchronized ovulation or synchronized estrus. Journal of Dairy Science, v.80, p.295-300, 1997. 
SENGER, P. L. The estrus detection problem: new concepts, technologies and possibilities. Journal of Dairy Science, v. 77, n. 9, p. 2745-2753, 1994. Disponível em: < http://www.dairy-science.org/cgi/reprint/77/9/2745>. Acesso em: 15 mai. 2010.

ZOCCAL, R. Rebanho de vacas ordenhadas, produção de leite e produtividade do rebanho no Brasil. In: EMBRAPA gado de leite. Juiz de Fora, [1997]. Disponível em:

< $\mathrm{HTTP}: / /$ www.cnpgl.embrapa.br>. Acesso em 27 abr. 2010. 